\title{
Event-driven NN adaptive fixed-time control for nonlinear systems with guaranteed performance
}

\author{
Xiaona Song • Peng Sun · Shuai Song
}

Received: date / Accepted: date

\begin{abstract}
This article investigates the adaptive neural network fixed-time tracking control for a class of strict-feedback nonlinear systems with prescribed performance demands, in which radial basis function neural network (RBFNN) is utilized to approximate the unknown items. First, an improved fractionalorder dynamic surface control (FODSC) technique is incorporated to address the issue of the iterative derivation, where a fractional-order filter is adopted to improve the filter performance. What's more, the error compensation signal is established to remove the impact of filter error. Furthermore, a fixed-time adaptive event-triggered controller is constructed to reduce the communication burden, where the Zeno-behavior can also be excluded. Stability results prove that the designed controller not only guarantees all the signals of the closedloop systems (CLS) are practically fixed-time bounded, but also the tracking error can be regulated to a predefined boundary. Finally, the feasibility and superiority of the designed control algorithm are verified by two simulation examples.
\end{abstract}

Keywords Event-triggered mechanism - fixed-time control · prescribed performance $\cdot$ fractional-order dynamic surface control - radial basis function neural network

\section{Introduction}

Adaptive backstepping control has been paid considerable attention during the past decades [1-3], where it is assumed that the nonlinear terms are known or bounded. However, the fact is that there are mostly unknown nonlinear functions in real circumstances. To this end, the fuzzy logic systems (FLSs) or the neural networks (NN) have been universally adopted to approximate the

X. Song, P. Sun, and S. Song

School of Information Engineering, Henan University of Science and Technology (E-mail: xiaona_97@163.com ) 
unknown nonlinear items [4-6]. Nevertheless, the issue of "explosion of complexity", which is generated by the iterative differentiation of virtual control signal can not be avoided. Consequently, the dynamic surface control (DSC) approach [7] was employed, where a first-order filter was incorporated into each stage within the backstepping design framework. Simultaneously, the adaptive NN control method was further combined with DSC to eradicate the effect of uncertain nonlinearities in $[8,9]$, but the filter error between the virtual control function and filter output is not fully considered. To tackle the above problems, a command filtered backstepping controller has been put forward in [10,11] via constructing the error compensation signals. Since then, an adaptive command filter quantized control method is developed for parametric nonlinear systems in [12]. In [13], on account of the command filtered backstepping controller, an adaptive neural control has emerged for nonlinear systems. In [14], command filtered-based adaptive controller is studied for nonlinear time-delay systems. It is worth emphasizing that the above-proposed controller based on command filtered backstepping control scheme only achieves asymptotic stability. However, in the case of practical applications, the requirement of finite-time convergence needs to be satisfied.

It is noteworthy that the finite-time control method guarantees the tracking errors converge quickly to the equilibrium point. Therefore, an increasing number of finite-time backstepping control methods have been presented gradually, see typically [15-17]. For example, command filter-based finite-time control framework was established for nonlinear systems in [15]. In [17], event-drivenbased adaptive finite-time control design was studied for uncertain nonlinear systems against event-trigger error. However, among the aforesaid problem, the settling time is closely associated with the initial values, which becomes unavailable when the initial values are far from the equilibrium point. Thus, the fixed-time stability problem was first proposed in [18], which forecasted settling time is independent of the initial conditions. In [19], an adaptive practical fixed-time tracking algorithm was explored for strict-feedback nonlinear systems. Furthermore, [20] introduced a fixed-time adaptive control for uncertain nonlinear systems.

Besides, many attempts have been devoted to coping with the control issue of nonlinear systems with performance constraints, since some requirements of performance behavior that convergence rate, maximum overshoot, and antiinterference capability arise in many engineering. For instance, [21] proposed the prescribed performance control strategy to conquer the performance constraints for the multiple-input multiple-output (MIMO) nonlinear systems. In [22], the adaptive fuzzy control method is developed for nonlinear system$\mathrm{s}$ with non-triangular structure. As an improvement, an adaptive finite-time control is settled out for stochastic feedback nonlinear systems in [23]. Then a natural question arises that the above results for nonlinear control problems without taking the network resource into account.

The control signal generated by the time-triggered control strategy $[24,25]$ will be splashed into the system at each sample time, whether it is demanded or not. Therefore, it may waste a large number of network resources. To tackle 
this problem, an approach named event-triggered control mechanism is used to replace the time-triggered. Recently, [26] has proposed an event-based sliding mode control scheme for active vehicle syspension systems. What's more, [27] has designed an event-triggered controller for nonlinear systems with full state constraints. In [28], by utilizing the event-triggered and prescribed performance, a robust adaptive finite-time control is addressed for the nonlinear system. However, to the best of our knowledge, the event-based adaptive prescribed performance fixed-time tracking control issue for the nonlinear system has not yet been fully studied in the framework of the FODSC.

Aiming at the above observations and discussions, in this work, we present an event-based adaptive NN fixed-time tracking control scheme for a class of strict-feedback nonlinear systems with prescribed performance demand by using an improved FODSC technique. The main contributions of this work are as follows:

1. By introducing a modified error compensation mechanism, we investigate an event-based adaptive fixed-time NN control method for strict-feedback nonlinear systems with prescribed performance demands, where the tracking error convergence toward prescribed range in the fixed-time interval. Additionally, the communication burden and filter errors are effectively decreased simultaneously.

2. Different from the existing integer-order (IO) filter results in [13, 14, 29], the main advantage of our proposed FODSC strategy is that "the explosion of complexity" problems is not only considerably circumvented, but also the filter performance is effectively enhanced. Consequently, it may be more generally suitable for practical engineering.

3. Based on the approximation abilities of neural networks $(\mathrm{NN})$, a fixedtime adaptive controller is established in this paper. Theoretical results prove that all the states of the CLS are practically fixed-time bounded, the system output can track the given reference signal, and settling time is not dependent on the initial values. As a result, the proposed controller provides a more efficient solution for solving practical tracking control problems than [30-32].

\section{Problem Formulation}

\subsection{System Description}

Consider the following uncertain nonlinear system:

$$
\left\{\begin{array}{l}
\dot{x}_{i}=x_{i+1}+f_{i}\left(\bar{x}_{i}\right), \\
\dot{x}_{n}=u+f_{n}(x), \\
y=x_{1},
\end{array}\right.
$$

where $\bar{x}_{i}=\left[x_{1}, \ldots, x_{i}\right]^{\mathrm{T}} \in R^{i}, i=1, \ldots, n-1$ and $x=\bar{x}_{n}=\left[x_{1}, \ldots, x_{n}\right]^{\mathrm{T}} \in R^{n}$ is the state vector, $y \in R$ is the output of the system, $f_{i}\left(\bar{x}_{i}\right): R^{i} \rightarrow R, i=$ $1, \ldots, n$, are unknown continuous functions, $u \in R$ is the control input. 
Assumption 1. The target signal $y_{d}$ and its first-order derivative $\dot{y}_{d}$ are bounded satisfying $\left|y_{d}\right| \leq y_{0},\left|\dot{y}_{d}\right| \leq y_{0}$.

To simplify the fixed-time control design, some critical assumptions, definitions, and mechanical lemmas are listed.

Definition 1 [32] The nonlinear system is considered in the following form

$$
\dot{x}(t)=f(t, x), x(0)=x_{0},
$$

where $x \in R^{n}$ represents the state vector and $f(\cdot)$ denotes the function with $f(0,0)=0$. Assuming that there is a settling time $T_{s}\left(\Psi, x_{0}\right)$ and a constant $\Psi$ satisfying $\|x(t)\|<\Psi$. For $\forall t \geq T_{s}\left(\Psi, x_{0}\right), \dot{x}(t)=f(t, x)$ is practically finitetime stable.

Definition 2 [18] Assume system $\dot{x}(t)=f(t, x)$ is finite-time stable. If there exists a constant $T_{\max }>0$ satisfying $T_{s}\left(\Psi, x_{0}\right) \leq T_{\max }, \dot{x}(t)=f(t, x)$ is described as practically fixed-time stable, which is independent of the initial variables.

Definition 3 [33] The Riemann-Liouville fractional derivative of order $q$ of a continuous function $h(t)$ is described as

$$
D^{q} h(t)=\frac{d^{n}}{d t^{n}}\left[\frac{1}{\mathrm{H}(n-q)} \int_{t_{0}}^{t} \frac{h(o)}{(t-o)^{q+1-n}} d o\right],
$$

where $n$ denotes an integer and satisfies $n-1<q<n$.

Lemma 1 [18] Consider $V(x)$ as a positive definite function satisfying the inequality as follows

$$
\dot{V}(x) \leq-\left(\phi_{1} V(x)^{\alpha}+\phi_{2} V(x)^{\beta}\right)^{\mathrm{I}}+\rho,
$$

where $\phi_{1}, \phi_{2}, \alpha, \beta$ and I are the positive numbers with $0<\alpha \mathrm{I}<1,1<\beta \mathrm{I}<\infty$ and $\rho>0$. Then, the origin $x=0$ of the system is practically fixed-time stable. Furthermore, the following inequality

$$
\left\{\lim _{t \rightarrow T_{l}} x \mid V(x) \leq \min \left\{\phi_{1}^{-\frac{1}{\alpha}}\left(\frac{\rho}{1-\varpi^{\mathrm{I}}}\right)^{\frac{1}{\alpha \mathrm{I}}}, \phi_{2}{ }^{-\frac{1}{\beta}}\left(\frac{\rho}{1-\varpi^{\mathrm{I}}}\right)^{\frac{1}{\beta 1}}\right\}\right\},
$$

is applied to indicate the boundary set of the system which has $0<\omega \leq 1$. The settling time is approximated by

$$
T_{l} \leq \frac{1}{\phi_{1}^{\mathrm{I}} \omega^{\mathrm{I}}(1-\alpha \mathrm{I})}+\frac{1}{\phi_{2}^{\mathrm{I}} \omega^{\mathrm{I}}(\beta \mathrm{I}-1)} .
$$

Remark 1 Similar to [34], to facilitate the derivation, the corresponding parameters are chosen as follows: $\alpha=\frac{3}{4}, \beta=2, \mathrm{I}=1$.

Lemma 2 [35] For a sufficient large constant $m$, unknown function $F(z)$ over a compact set $\Omega_{z} \subset R^{m}$ can be approximated by the following radial basis function neural network (RBFNN) satisfying

$$
F(z)=W^{* T} S(z)+\delta(z),
$$


where $W^{*}$ represents an optimal weight vector satisfying

$$
W^{*}=\arg \min _{W \in R^{m}}\left\{\sup _{z \in \Omega_{z}}\left|F(z)-W^{T} S(z)\right|\right\},
$$

where $\delta(z)$ represents an approximation error with $|\delta(z)| \leq \varepsilon$ and $\forall \varepsilon>0$, $W=\left[W_{1}, \ldots, W_{m}\right]^{T} \in R^{m}$ represents the weight vector with $m$ is the number of RBFNN nodes; $S(z)=\left[s_{1}(z), \ldots, s_{m}(z)\right]^{T}$ is the basis function vector satisfying

$$
s_{i}(z)=\exp \left[-\frac{\left(z-c_{i}\right)^{T}\left(z-c_{i}\right)}{b_{i}^{2}}\right],
$$

where $c_{i}=\left[c_{i 1}, c_{i 2}, \ldots, c_{i k}\right]^{T}(i=1, \ldots, m)$ denotes the center of the receptive field, and $b_{i}>0$ denotes the width of the basis function.

Lemma 3 [36] For any real variables $x_{1}, y_{2}$ and any real numbers $k_{1}, k_{2}, \mathrm{~B}$, there holds

$$
\left|x_{1}\right|^{k_{1}}\left|y_{2}\right|^{k_{2}} \leq \frac{k_{1}}{k_{1}+k_{2}} \mathrm{~B}\left|x_{1}\right|^{k_{1}+k_{2}}+\frac{k_{2}}{k_{1}+k_{2}} \mathrm{~B}^{\frac{-k_{1}}{k_{2}}}\left|y_{2}\right|^{k_{1}+k_{2}} .
$$

Lemma 4 [37] For $0<p<1, \mathrm{H}_{i} \in R, i=1, \ldots, n$, , one has

$$
\left(\left|\mathrm{H}_{1}\right|+\cdots+\left|\mathrm{H}_{n}\right|\right)^{p} \leq\left|\mathrm{H}_{1}\right|^{p}+\cdots+\left|\mathrm{H}_{n}\right|^{p} .
$$

Lemma 5 [38] For $\zeta_{i} \in R, i=1, \ldots, N$, then

$$
\begin{aligned}
& \left(\sum_{i=1}^{N}\left|\zeta_{i}\right|\right)^{\iota} \leq \sum_{i=1}^{N}\left|\zeta_{i}\right|^{\iota}, 0<\iota \leq 1, \\
& N^{1-\iota}\left(\sum_{i=1}^{N}\left|\zeta_{i}\right|\right)^{\iota} \leq \sum_{i=1}^{N}\left|\zeta_{i}\right|^{\iota}, \iota>1 .
\end{aligned}
$$

\subsection{Event-Triggered Mechanism}

In this section, based on relative-threshold event-triggered control scheme is designed as below [39]:

$$
\begin{aligned}
& v=-(1+\tau)\left(\alpha_{n} \tanh \left(\frac{\varsigma_{n} \alpha_{n}}{\rho}\right)+\mu_{1} \tanh \left(\frac{\varsigma_{n} \mu_{1}}{\rho}\right)\right), \\
& u(t)=v\left(t_{k}\right), \forall t \in\left[t_{k}, t_{k+1}\right), \\
& t_{k+1}=\inf \left\{t \in R \| \mathrm{P}|\geq \tau| u \mid+\mu_{2}\right\},
\end{aligned}
$$

where $\varsigma_{n}, \alpha_{n}$ will be designed later, the event-triggered error $\mathrm{P}=v-u, 0<$ $\tau<1, \rho>0, \mu_{1}>0$ and $\mu_{2}>0$ satisfying $\mu_{1}>\frac{\mu_{2}}{1-\tau} \cdot t_{k}$ represents input update time. Note that, for the time $t \in\left[t_{k}, t_{k+1}\right), u$ views as a constant $v\left(t_{k}\right)$. When (4) is activated, the instant is denoted as $t_{k+1}$ and control signals $u\left(t_{k+1}\right)$ 
can be utilized in the system. Thus, we can find the parameters satisfying $\ell_{1}, \ell_{2}$, the following equation holds

$$
v=\left(1+\ell_{1} \tau\right) u+\ell_{2} \mu_{2},
$$

where $\left|\ell_{1}\right| \leq 1$ and $\left|\ell_{2}\right| \leq 1$. Therefore, one has

$$
u=\frac{v-\ell_{2} \mu_{2}}{1+\ell_{1} \tau} .
$$

\subsection{Prescribed Performance}

The tracking error $j_{1}$ in this article is constrained to the following ranges

$$
\Omega_{j 1}=\left\{j_{1} \in R \mid-\sigma<j_{1}<\sigma\right\},
$$

where $\sigma$ denotes desired performance function and the tracking error is depicted as $j_{1}=y-y_{d} . \sigma$ can be defined as

$$
\sigma=\left\{\begin{array}{l}
\left(\bar{\sigma}_{0}-a \kappa t\right)^{\frac{1}{a}}+\sigma_{T_{0}}, 0 \leq t \leq T_{0}, \\
\sigma_{T_{0}}
\end{array}\right.
$$

where $a=\frac{\mathrm{E}_{1}}{\mathrm{E}_{2}} \in(0,1)$ with $\mathrm{E}_{1}$ and $\mathrm{E}_{2}$ are specified as positive odd integers. The convergence rate, the ultimate limits of performance function and the convergence time are described as $a, \sigma_{T_{0}}>0$ and $T_{0}>0$, which need to be preset. When the above three parameters and initial state $\sigma_{0}$ are confirmed, the rest of the parameters $\bar{\sigma}_{0}, \kappa$ can be settled such that all the parameters must comply with $T_{0}=\frac{\bar{\sigma}_{0}}{a \kappa}$ and $\bar{\sigma}_{0}^{\frac{1}{a}}+\sigma_{T_{0}}=\sigma(0)$.

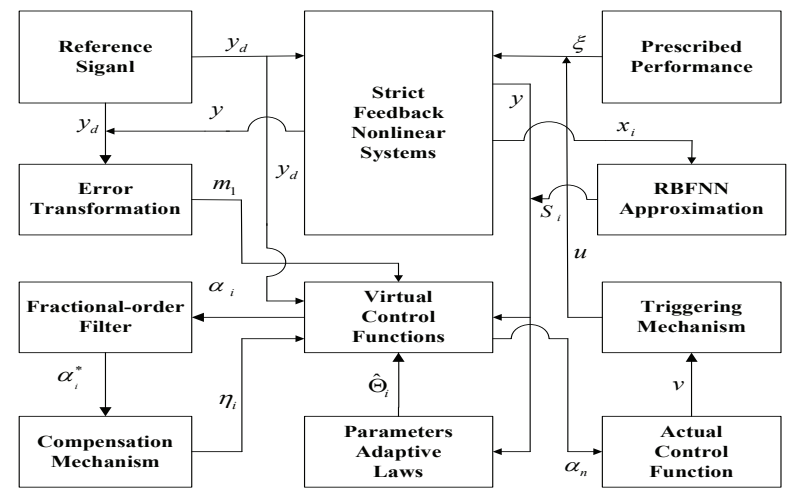

Fig. 1: The flow diagram of FODSC-based fixed-time controller.

Control objective: this work aims to construct a fixed-time adaptive control scheme such that the output signal $y$ can track given reference signal $y_{d}$; all the signals in the CLS are practically fixed-time bounded; the tracking 
error $j_{1}$ can strictly converge to an arbitrary predefined boundary in a fixedtime period. What's more, the Zeno phenomenon is avoided. The flow diagram of the designed algorithm is displayed in Fig. 1.

\section{Main results}

In this section, an event-triggered adaptive $\mathrm{NN}$ fixed-time control method will be presented by integrating with a modified FODSC approach.

\subsection{State Transformation}

To facilitate controller design, the following coordinate transformation is introduced [42]

$$
m_{1}=\tan \left(\frac{\pi j_{1}}{2 \sigma}\right), j_{1}(0)<\sigma(0)
$$

from (9) that

$$
j_{1}=\frac{2}{\pi} \sigma \arctan \left(m_{1}\right)
$$

Taking the derivative of $j_{1}$ gives

$$
\dot{j}_{1}=\frac{2}{\pi} \dot{\sigma} \arctan \left(m_{1}\right)+\frac{2}{\pi} \sigma \frac{\dot{m}_{1}}{1+m_{1}^{2}}=x_{2}+f_{1}\left(x_{1}\right)-\dot{y}_{d}
$$

then, (11) can be expressed as

$$
\dot{m}_{1}=d_{1} x_{2}+d_{1} f_{1}\left(x_{1}\right)-d_{1} \dot{y}_{d}-\frac{2}{\pi} \dot{\sigma} d_{1} \arctan \left(m_{1}\right)
$$

where $d_{1}=\frac{\pi\left(1+m_{1}^{2}\right)}{2 \sigma}>0$.

Remark 2 The purpose of introducing the state transformation (9) is to convert the restrained error $j_{1}$ to an unrestrained state $m_{1}$. Then, it can be speculated from (9) that the boundedness of $m_{1}$ implies that $-\sigma<j_{1}<\sigma$. Furthermore, $m_{1} \rightarrow 0 \Rightarrow j_{1} \rightarrow 0$. Therefore, the next assignment is to assure the boundedness and the convergence of $m_{1}$. 
3.2 FODSC-Based Fixed-time Controller

To facilitate controller design, the corresponding coordinate transformations are defined as below:

$$
\left\{\begin{array}{l}
z_{1}=m_{1}, \\
z_{i}=x_{i}-\alpha_{i}^{*}, i=2, \ldots, n,
\end{array}\right.
$$

in which $\alpha_{i}^{*}$ denotes the output of the following FO filter with the virtual control signal $\alpha_{i}$ as the input:

$$
\beta_{i} D^{q} \alpha_{i}^{*}+\alpha_{i}^{*}=\alpha_{i}, \alpha_{i}(0)=\alpha_{i}^{*}(0),
$$

where $D^{q}$ represents the fractional operator with $0<q<1$ and $\beta_{i}>0$ is a time constant.

Remark 3 Due to the data memory and hereditary characteristics of fractional calculus, it has been acquired that FO control improves the degree of freedom for achieving higher control accuracy as opposed to IO [40,41]. Thus, we attempt to develop a FODSC-based adaptive fixed-time method for the system (1) in this paper. Different from the most of existing results $[13,14,29]$, the proposed FO filter not only avoids the computational complexity caused by the traditional backstepping algorithm but also incorporates the FO characteristic into the filter design.

The compensation error $\varsigma_{i}$ is defined as follow:

$$
\varsigma_{i}=z_{i}-\eta_{i}, i=1, \ldots, n .
$$

To compensate for the filter error between the virtual control signal and the filter output, the compensation system $\eta_{i}$ is designed as

$$
\left\{\begin{array}{l}
\dot{\eta}_{1}=-k_{11} \eta_{1}^{\frac{1}{2}}-k_{12} \eta_{1}^{3}+d_{1}\left(\alpha_{2}^{*}-\alpha_{2}\right)+d_{1} \eta_{2} \\
\dot{\eta}_{i}=-k_{i 1} \eta_{i}^{\frac{1}{2}}-k_{i 2} \eta_{i}^{3}-d_{i-1} \eta_{i-1}+d_{i} \eta_{i+1}+d_{i}\left(\alpha_{i+1}^{*}-\alpha_{i+1}\right) \\
\dot{\eta}_{n}=-k_{n 1} \eta_{n}^{\frac{1}{2}}-k_{n 2} \eta_{n}^{3}-d_{n-1} \eta_{n-1}
\end{array}\right.
$$

with $\eta_{i}(0)=0, d_{l}=1(l=2, \ldots, n), k_{i 1}>0, k_{i 2}>0$ are design parameters.

Step 1. From (12)-(13) and calculating the first-order derivative of $z_{1}$, one has

$$
\begin{aligned}
\dot{z}_{1}= & d_{1} z_{2}+d_{1}\left(\alpha_{2}^{*}-\alpha_{2}\right)+d_{1} \alpha_{2}+d_{1} f_{1}\left(x_{1}\right) \\
& -d_{1} \dot{y}_{d}-\frac{2}{\pi} d_{1} \dot{\sigma} \arctan \left(m_{1}\right) .
\end{aligned}
$$

To overcome the influences of errors $\alpha_{2}^{*}-\alpha_{2}$, the compensating signal $\eta_{2}$ is introduced as follows:

$$
\dot{\eta}_{1}=-k_{11} \eta_{1}^{\frac{1}{2}}-k_{12} \eta_{1}^{3}+d_{1} \eta_{2}+d_{1}\left(\alpha_{2}^{*}-\alpha_{2}\right),
$$

with $\eta_{1}(0)=0$. Combining (15) with (17) and (18) holds

$$
\dot{\varsigma}_{1}=d_{1} \varsigma_{2}+d_{1} \alpha_{2}+k_{11} \eta_{1}^{\frac{1}{2}}+d_{1} f_{1}\left(x_{1}\right)-d_{1} \dot{y}_{d}
$$




$$
-\frac{2}{\pi} d_{1} \dot{\sigma} \arctan \left(m_{1}\right)+k_{12} \eta_{1}^{3}
$$

Now, choose a Lyapunov function candidate as:

$$
V_{1}=\frac{1}{2} \varsigma_{1}^{2}+\frac{1}{2 \bar{c}_{1}} \tilde{\Theta}_{1}^{2}
$$

where $\tilde{\Theta}=\Theta-\hat{\Theta}$ represents the estimation error of the unknown parameter, the time differentiation of $V_{1}$ satisfies

$$
\begin{aligned}
\dot{V}_{1}= & d_{1} \varsigma_{1}\left(\varsigma_{2}+\alpha_{2}+f_{1}\left(x_{1}\right)-\frac{2}{\pi} \dot{\sigma} \arctan \left(m_{1}\right)\right. \\
& \left.+\frac{k_{11}}{d_{1}} \eta_{1}^{\frac{1}{2}}+\frac{k_{12}}{d_{1}} \eta_{1}^{3}-\dot{y}_{d}\right)-\frac{1}{\bar{c}_{1}} \tilde{\Theta}_{1} \dot{\hat{\Theta}}_{1} .
\end{aligned}
$$

Then, the packaged function is set as $\Lambda_{1}=f_{1}\left(x_{1}\right)$.

In view of Lemma 2 , a RBFNN $W_{1}^{* \mathrm{~T}} S_{1}(z)$ is applied to approximate the unknown term $\Lambda_{1}$. For $\forall \varepsilon_{1}>0$, one has

$$
\Lambda_{1}=W_{1}^{* \mathrm{~T}} S_{1}(z)+\delta_{1}(z),
$$

where $\left|\delta_{1}(z)\right|<\varepsilon_{1}$ represents the approximation error.

Furthermore, in accordance with Lemma 3 and $\Theta_{i}=\max _{1 \leq i \leq n}\left\{\left\|W_{i}^{*}\right\|^{2}\right\}$, it can be acquired

$$
\begin{aligned}
\varsigma_{1} d_{1} W_{1}^{* \mathrm{~T}} S_{1}(z) & \leq \frac{1}{2 a_{11}} \Theta_{1}\left\|S_{1}\right\|^{2} d_{1}^{2} \varsigma_{1}^{2}+\frac{a_{11}}{2}, \\
\varsigma_{1} d_{1} \delta_{1}(z) & \leq \frac{1}{2 a_{12}} d_{1}^{2} \varsigma_{1}^{2}+\frac{a_{12}}{2} \varepsilon_{1}^{2} .
\end{aligned}
$$

By plugging (22)-(23) into (21), results in

$$
\begin{aligned}
\dot{V}_{1} \leq & \varsigma_{1} d_{1} \varsigma_{2}+\varsigma_{1} d_{1}\left(\alpha_{2}+\frac{d_{1}}{2 a_{11}} \Theta_{1}\left\|S_{1}\right\|^{2} \varsigma_{1}+\frac{k_{11}}{d_{1}} \eta_{1}^{\frac{1}{2}}\right. \\
& \left.+\frac{1}{2 a_{12}} d_{1} \varsigma_{1}-\frac{2}{\pi} \dot{\sigma} \arctan \left(m_{1}\right)+\frac{k_{12}}{d_{1}} \eta_{1}^{3}-\dot{y}_{d}\right) \\
& +\frac{1}{2}\left(a_{11}+a_{12} \varepsilon_{1}^{2}\right)-\frac{1}{\bar{c}_{1}} \tilde{\Theta}_{1} \dot{\hat{\Theta}}_{1} .
\end{aligned}
$$

To fulfill the control objective, a fixed-time virtual control signal $\alpha_{2}$ and the adaptive laws $\dot{\hat{\Theta}}_{1}$ are designed as

$$
\begin{aligned}
\alpha_{2}= & -\frac{d_{1}}{2 a_{11}} \hat{\Theta}_{1}\left\|S_{1}\right\|^{2} \varsigma_{1}-\frac{k_{11}}{d_{1}} z_{1}^{\frac{1}{2}}-\frac{k_{12}}{d_{1}} z_{1}^{3}-\frac{1}{2 a_{12}} d_{1} \varsigma_{1} \\
& +\frac{2}{\pi} \dot{\sigma} \arctan \left(m_{1}\right)+\dot{y}_{d}, \\
\dot{\hat{\Theta}}_{1}= & \frac{d_{1}^{2} \bar{c}_{1}}{2 a_{11}}\left\|S_{1}\right\|^{2} \varsigma_{1}^{2}-\bar{r}_{1} \hat{\Theta}_{1}-\frac{\xi_{1}}{\bar{c}_{1}} \hat{\Theta}_{1}^{3} .
\end{aligned}
$$


Substituting (25)-(26) into (24) yields

$$
\dot{V}_{1} \leq d_{1} \varsigma_{1} \varsigma_{2}-k_{11}\left(\varsigma_{1}^{2}\right)^{\frac{3}{4}}-k_{12}\left(\varsigma_{1}^{2}\right)^{2}+\frac{\bar{r}_{1}}{\bar{c}_{1}} \hat{\Theta}_{1} \tilde{\Theta}_{1}+\frac{\xi_{1}}{\bar{c}_{1}^{2}} \tilde{\Theta}_{1} \hat{\Theta}_{1}^{3}+\chi_{1},
$$

where $\chi_{1}=\frac{1}{2}\left(a_{11}+a_{12} \varepsilon_{1}^{2}\right)$.

Moreover, it is assumed that $\alpha_{k}(3 \leq k \leq n)$ can be represented as follows

$$
\begin{aligned}
\dot{V}_{k-1} \leq & -\sum_{l=1}^{k-1} k_{l 1}\left(\varsigma_{l}^{2}\right)^{\frac{3}{4}}-\sum_{l=1}^{k-1} k_{l 2}\left(\varsigma_{l}^{2}\right)^{2}+\sum_{l=1}^{k-1} \frac{\bar{r}_{l}}{\bar{c}_{l}} \tilde{\Theta}_{l} \hat{\Theta}_{l} \\
& +\sum_{l=1}^{k-1} \frac{\xi_{l}}{\bar{c}_{l}^{2}} \tilde{\Theta}_{l} \hat{\Theta}_{l}^{3}+\chi_{k-1}+\varsigma_{k-1} d_{k-1} \varsigma_{k} .
\end{aligned}
$$

Step $k$. By (1) and (13), it can be deduced that

$$
\dot{z}_{\mathrm{k}}=z_{\mathrm{k}+1}+\alpha_{k+1}^{*}-\alpha_{k+1}+\alpha_{k+1}+f_{k}\left(x_{k}\right)-\dot{\alpha}_{k}^{*} .
$$

It follows from (15) and (16) that

$$
\dot{\varsigma}_{k}=z_{\mathrm{k}+1}+\alpha_{k+1}+f_{k}\left(x_{k}\right)-\dot{\alpha}_{k}^{*}+k_{k 1} \eta_{k}^{\frac{1}{2}}+k_{k 2} \eta_{k}^{3}+\eta_{k-1}-\eta_{k+1} .
$$

Consider Lyapunov function $V_{k}=V_{k-1}+\frac{1}{2} \varsigma_{k}^{2}+\frac{1}{2 \bar{c}_{k}} \tilde{\Theta}_{k}^{2}$ and calculating its time derivative, it can be obtained that

$$
\begin{aligned}
\dot{V}_{k} \leq & \varsigma_{k}\left(\alpha_{k+1}+f_{k}\left(x_{k}\right)-\dot{\alpha}_{k}^{*}+k_{k 1} \eta_{k}^{\frac{1}{2}}+k_{k 2} \eta_{k}^{3}+\eta_{k-1}\right) \\
& +\varsigma_{k} \varsigma_{k+1}-\frac{1}{\bar{c}_{k}} \tilde{\Theta}_{k} \dot{\hat{\Theta}}_{k}+\dot{V}_{k-1} .
\end{aligned}
$$

By using RBFNN to approximate the nonlinear term $\Lambda_{k}=f_{k}\left(x_{k}\right)-$ $\dot{\alpha}_{k}^{*}+\varsigma_{k-1}$, one has $W_{k}^{* \mathrm{~T}} S_{k}(z)+\delta_{k}(z)$, where $\left|\delta_{k}(z)\right| \leq \varepsilon_{k}$ is the approximation error, for any given $\varepsilon_{k}>0$.

In light of Lemma 3, it can be expressed as

$$
\begin{aligned}
\varsigma_{k} W_{k}^{* \mathrm{~T}} S_{1}(z) & \leq \frac{1}{2 a_{k 1}} \Theta_{k}\left\|S_{k}\right\|^{2} \varsigma_{k}^{2}+\frac{a_{k 1}}{2}, \\
\varsigma_{k} \delta_{k}(z) & \leq \frac{1}{2 a_{k 2}} \varsigma_{k}^{2}+\frac{a_{k 2}}{2} \varepsilon_{k}^{2} .
\end{aligned}
$$

Thus, (31) can be further written as

$$
\begin{aligned}
\dot{V}_{k} \leq & -\sum_{l=1}^{k-1} k_{l 1}\left(\varsigma_{l}^{2}\right)^{\frac{3}{4}}-\sum_{l=1}^{k-1} k_{l 2}\left(\varsigma_{l}^{2}\right)^{2}+\sum_{l=1}^{k-1} \frac{\bar{r}_{l}}{\bar{c}_{l}} \tilde{\Theta}_{l} \hat{\Theta}_{l}+\sum_{l=1}^{k-1} \frac{\xi_{l}}{\bar{c}_{l}^{2}} \tilde{\Theta}_{l} \hat{\Theta}_{l}^{3} \\
& +\varsigma_{k}\left(\alpha_{k+1}+k_{k 1} \eta_{k}^{\frac{1}{2}}+k_{k 2} \eta_{k}^{3}+\frac{1}{2 a_{k 2}} \varsigma_{k}+\eta_{k-1}\right)+\chi_{k-1} \\
& -\frac{1}{\bar{c}_{k}} \tilde{\Theta}_{k} \dot{\hat{\Theta}}_{k}+\varsigma_{k} \varsigma_{k+1}+\frac{1}{2 a_{k 1}} \Theta_{\mathrm{k}}\left\|S_{k}\right\|^{2} \varsigma_{k}^{2}+\frac{1}{2}\left(a_{k 1}+a_{k 2} \varepsilon_{k}^{2}\right) .
\end{aligned}
$$


Design the virtual controller $\alpha_{k+1}$ and the adaptive laws $\dot{\hat{\Theta}}_{k}$ as follows:

$$
\begin{aligned}
& \alpha_{k+1}=-\frac{1}{2 a_{k 1}} \varsigma_{k} \hat{\Theta}_{k}\left\|S_{k}\right\|^{2}-k_{k 1}\left(z_{k}\right)^{\frac{1}{2}}-k_{k 2}\left(z_{k}\right)^{3}-\frac{1}{2 a_{k 2}} \varsigma_{k}-\eta_{k-1}, \\
& \dot{\hat{\Theta}}_{k}=\frac{\bar{c}_{k}}{2 a_{k 1}}\left\|S_{k}\right\|^{2} \varsigma_{k}^{2}-\bar{r}_{k} \hat{\Theta}_{k}-\frac{\xi_{k}}{\bar{c}_{k}} \hat{\Theta}_{k}^{3} .
\end{aligned}
$$

Then, (34) can be represented as

$$
\begin{aligned}
\dot{V}_{k} \leq & -\sum_{l=1}^{k} k_{l 1}\left(\varsigma_{l}^{2}\right)^{\frac{3}{4}}-\sum_{l=1}^{k} k_{l 2}\left(\varsigma_{l}^{2}\right)^{2}+\sum_{l=1}^{k} \frac{\bar{r}_{l}}{\bar{c}_{l}} \hat{\Theta}_{l} \tilde{\Theta}_{l} \\
& +\sum_{l=1}^{k} \frac{\xi_{l}}{\bar{c}_{l}^{2}} \tilde{\Theta}_{l} \hat{\Theta}_{l}^{3}+\chi_{k}+\varsigma_{k} \varsigma_{k+1}
\end{aligned}
$$

where $\chi_{k}=\chi_{k-1}+\frac{1}{2}\left(a_{k 1}+a_{k 2} \varepsilon_{k}^{2}\right)$.

Step $n$. As is the same case of step $\mathrm{k}$, one has

$$
\dot{z}_{\mathrm{n}}=\dot{x}_{n}-\dot{\alpha}_{n}^{*}=u+f_{n}(x)-\dot{\alpha}_{n}^{*},
$$

the corresponding compensating signal $\eta_{n}$ is introduced:

$$
\dot{\eta}_{n}=-k_{n 1} \eta_{n}^{\frac{1}{2}}-k_{n 2} \eta_{n}^{3}-d_{n-1} \eta_{n-1},
$$

with $\eta_{n}(0)=0$. From (38)-(39), one has

$$
\dot{\varsigma}_{n}=u+f_{n}(x)-\dot{\alpha}_{n}^{*}+k_{n 1} \eta_{n}^{\frac{1}{2}}+k_{n 2} \eta_{n}^{3}+d_{n-1} \eta_{n-1} .
$$

Construct a Lyapunov function candidate as follows:

$$
V_{n}=\frac{1}{2} \varsigma_{n}^{2}+\frac{1}{2 \bar{c}_{n}} \tilde{\Theta}_{n}^{2}+V_{n-1},
$$

its derivative is given as

$$
\begin{aligned}
\dot{V}_{n} \leq & \varsigma_{n} \\
& \left(u+f_{n}(x)-\alpha_{n}^{*}+k_{n 1} \eta_{n}^{\frac{1}{2}}+k_{n 2} \eta_{n}^{3}+d_{n-1} \eta_{n-1}\right) \\
& -\sum_{l=1}^{n-1} k_{l 1}\left(\varsigma_{l}^{2}\right)^{\frac{3}{4}}-\sum_{l=1}^{n-1} k_{l 2}\left(\varsigma_{l}^{2}\right)^{2}+\sum_{l=1}^{n-1} \frac{\bar{r}_{l}}{\bar{c}_{l}} \hat{\Theta}_{l} \tilde{\Theta}_{l} \\
& +\sum_{l=1}^{n-1} \frac{\xi_{l}}{\bar{c}_{l}^{2}} \tilde{\Theta}_{l} \hat{\Theta}_{l}^{3}+\chi_{n-1}-\frac{1}{\bar{c}_{n}} \tilde{\Theta}_{n} \dot{\hat{\Theta}}_{n}+d_{n-1} \varsigma_{n-1} \varsigma_{n} .
\end{aligned}
$$

By setting $\Lambda_{n}=f_{n}(x)-\dot{\alpha}_{n}^{*}+d_{n-1} \varsigma_{n-1}$. For any given $\varepsilon_{n}>0$, this unknown function $\Lambda_{n}$ is able to be imitated as

$$
\Lambda_{n}=W_{n}^{* \mathrm{~T}} S_{n}(z)+\delta_{n}(z),
$$


where $\left|\delta_{n}(z)\right| \leq \varepsilon_{n}$ is the approximation error. Furthermore, one has

$$
\begin{aligned}
\varsigma_{n} W_{n}^{* \mathrm{~T}} S_{n}(z) & \leq \frac{1}{2 a_{n 1}} \Theta\left\|S_{n}\right\|^{2} \varsigma_{n}^{2}+\frac{a_{n 1}}{2}, \\
\varsigma_{n} \delta_{n}(z) & \leq \frac{1}{2 a_{n 2}} \varsigma_{n}^{2}+\frac{a_{n 2}}{2} \varepsilon_{n}^{2} .
\end{aligned}
$$

By substituting (43)-(44) into (42), one has

$$
\begin{aligned}
\dot{V}_{n} \leq & -\sum_{l=1}^{n-1} k_{l 1}\left(\varsigma_{l}^{2}\right)^{\frac{3}{4}}-\sum_{l=1}^{n-1} k_{l 2}\left(\varsigma_{l}^{2}\right)^{2}+\sum_{l=1}^{n-1} \frac{\bar{r}_{l}}{\bar{c}_{l}} \hat{\Theta}_{l} \tilde{\Theta}_{l}+\sum_{l=1}^{n-1} \frac{\xi_{l}}{\bar{c}_{l}^{2}} \tilde{\Theta}_{l} \hat{\Theta}_{l}^{3} \\
& -\frac{1}{\bar{c}_{n}} \tilde{\Theta}_{n} \dot{\hat{\Theta}}_{n}+\varsigma_{n}\left(u+\frac{1}{2 a_{n 2}} \varsigma_{n}+k_{n 1} \eta_{n}^{\frac{1}{2}}+k_{n 2} \eta_{n}^{3}+d_{n-1} \eta_{n-1}\right) \\
& +\frac{1}{2 a_{n 1}} \Theta_{n}\left\|S_{n}\right\|^{2} \varsigma_{n}^{2}+\chi_{n-1}+\frac{1}{2}\left(a_{n 1}+a_{n 2} \varepsilon_{n}^{2}\right) .
\end{aligned}
$$

Before constructing the event-triggered actual controller, the virtual control signal $\alpha_{n}$ and the adaptive laws $\dot{\hat{\Theta}}_{n}$ are designed as follows:

$$
\begin{aligned}
& \alpha_{n}=-\frac{1}{2 a_{n 1}} \varsigma_{n} \hat{\Theta}_{n}\left\|S_{n}\right\|^{2}-k_{n 1}\left(z_{n}\right)^{\frac{1}{2}}-k_{n 2}\left(z_{n}\right)^{3}-\frac{1}{2 a_{n 2}} \varsigma_{n}-d_{n-1} \eta_{n-1}, \\
& \dot{\hat{\Theta}}_{n}=\frac{\bar{c}_{n}}{2 a_{n 1}}\left\|S_{n}\right\|^{2} \varsigma_{n}^{2}-\bar{r}_{n} \hat{\Theta}_{n}-\frac{\xi_{n}}{\bar{c}_{n}} \hat{\Theta}_{n}^{3} .
\end{aligned}
$$

In the interval times $\left[t_{k}, t_{k+1}\right)$, and from (3), it can be obtained that $|v-u|<\tau|u|+\mu_{2}$. Considering (6) and (45)-(47), yields

$$
\begin{aligned}
\dot{V}_{n} \leq & -\sum_{l=1}^{n} k_{l 1}\left(\varsigma_{l}^{2}\right)^{\frac{3}{4}}-\sum_{l=1}^{n} k_{l 2}\left(\varsigma_{l}^{2}\right)^{2}-\varsigma_{n} \alpha_{n}-\frac{\varsigma_{n} \ell_{2} \mu_{2}}{1+\ell_{1} \tau} \\
& -\frac{1+\tau}{1+\ell_{1} \tau}\left(\varsigma_{n} \alpha_{n} \tanh \left(\frac{\varsigma_{n} \alpha_{n}}{\rho}\right)+\varsigma_{n} \mu_{1} \tanh \left(\frac{\varsigma_{n} \mu_{1}}{\rho}\right)\right) \\
& +\sum_{l=1}^{n} \frac{\bar{r}_{l}}{\bar{c}_{l}} \hat{\Theta}_{l} \tilde{\Theta}_{l}+\sum_{l=1}^{n} \frac{\xi_{l}}{\bar{c}_{l}^{2}} \tilde{\Theta}_{l} \hat{\Theta}_{l}^{3}+\chi_{n-1}+\frac{1}{2}\left(a_{n 1}+a_{n 2} \varepsilon_{n}^{2}\right) .
\end{aligned}
$$

Due to $0<1+\ell_{1} \tau<1+\tau$ and $-\frac{\varsigma_{n} \ell_{2} \mu_{2}}{1+\ell_{1} \tau} \leq\left|\frac{\varsigma_{n} \mu_{2}}{1-\tau}\right| \leq\left|\varsigma_{n} \mu_{1}\right|$, the inequality (48) can be rewritten as

$$
\begin{aligned}
\dot{V}_{n} \leq & -\sum_{l=1}^{n} k_{l 1}\left(\varsigma_{l}^{2}\right)^{\frac{3}{4}}-\sum_{l=1}^{n} k_{l 2}\left(\varsigma_{l}^{2}\right)^{2}+\left|\varsigma_{n} \alpha_{n}\right|+\left|\varsigma_{n} \mu_{1}\right| \\
& -\varsigma_{n} \alpha_{n} \tanh \left(\frac{\varsigma_{n} \alpha_{n}}{\rho}\right)-\varsigma_{n} \mu_{1} \tanh \left(\frac{\varsigma_{n} \mu_{1}}{\rho}\right)+\sum_{l=1}^{n} \frac{\bar{r}_{l}}{\bar{c}_{l}} \hat{\Theta}_{l} \tilde{\Theta}_{l} \\
& +\sum_{l=1}^{n} \frac{\xi_{l}}{\bar{c}_{l}^{2}} \tilde{\Theta}_{l} \hat{\Theta}_{l}^{3}+\chi_{n-1}+\frac{1}{2}\left(a_{n 1}+a_{n 2} \varepsilon_{n}^{2}\right) .
\end{aligned}
$$


Additionally, if $\psi \in R$ and $q>0$, the hyperbolic tangent function satisfies $0 \leq|\psi|-\psi \tanh \left(\frac{\psi}{\bar{q}}\right) \leq 0.2785 \bar{q}$, one obtains

$$
\dot{V}_{n} \leq+\sum_{l=1}^{n} \frac{\xi_{l}}{\bar{c}_{l}^{2}} \tilde{\Theta}_{l} \hat{\Theta}_{l}^{3}-\sum_{l=1}^{n} k_{l 2}\left(\varsigma_{l}^{2}\right)^{2}+\sum_{l=1}^{n} \frac{\bar{r}_{l}}{\bar{c}_{l}} \hat{\Theta}_{l} \tilde{\Theta}_{l}-\sum_{l=1}^{n} k_{l 1}\left(\varsigma_{l}^{2}\right)^{\frac{3}{4}}+\chi_{n}
$$

where $\chi_{n}=\chi_{n-1}+\frac{1}{2}\left(a_{n 1}+a_{n 2} \varepsilon_{n}^{2}\right)+0.557 \bar{q}$.

By defining $\left(\frac{1}{2}\right)^{\frac{3}{4}} \bar{\vartheta}_{1}=\min \left\{k_{11}, \ldots, k_{n 1}\right\},\left(\frac{1}{2}\right)^{2} \bar{\vartheta}_{2}=\min$ $\left\{k_{12}, \ldots, k_{n 2}\right\}$ and utilizing Lemma 4 and Lemma 5 , (50) can be confirmed the following form

$$
\begin{aligned}
& -\sum_{l=1}^{n} k_{l 1}\left(\varsigma_{l}^{2}\right)^{\frac{3}{4}} \leq-\bar{\vartheta}_{1} \sum_{l=1}^{n}\left(\frac{\varsigma_{l}^{2}}{2}\right)^{\frac{3}{4}} \leq-\bar{\vartheta}_{1}\left(\sum_{l=1}^{n} \frac{\varsigma_{l}^{2}}{2}\right)^{\frac{3}{4}}, \\
& -\sum_{l=1}^{n} k_{l 2}\left(\varsigma_{l}^{2}\right)^{2} \leq-\bar{\vartheta}_{2} \sum_{l=1}^{n}\left(\frac{\varsigma_{l}^{2}}{2}\right)^{2} \leq-\frac{\bar{\vartheta}_{2}}{n}\left(\sum_{l=1}^{n} \frac{\varsigma_{l}^{2}}{2}\right)^{2} .
\end{aligned}
$$

In the light of $\tilde{\Theta}_{l} \hat{\Theta}_{l} \leq-\frac{\tilde{\Theta}_{l}^{2}}{2}+\frac{\Theta_{l}^{2}}{2}$, one has

$$
\sum_{l=1}^{n} \frac{\bar{r}_{l}}{\bar{c}_{l}} \hat{\Theta}_{l} \tilde{\Theta}_{l} \leq-\sum_{l=1}^{n} \frac{\bar{r}_{l} \tilde{\Theta}_{l}^{2}}{2 \bar{c}_{l}}+\sum_{l=1}^{n} \frac{\bar{r}_{l} \Theta_{l}^{2}}{2 \bar{c}_{l}} .
$$

Thus, substituting (51), (52) and (53) into (50), one can obtain

$$
\begin{aligned}
\dot{V}_{n} \leq & -\bar{\vartheta}_{1}\left(\sum_{l=1}^{n} \frac{\varsigma_{l}^{2}}{2}\right)^{\frac{3}{4}}-\left(\sum_{l=1}^{n} \frac{\bar{r}_{l} \tilde{\Theta}_{l}^{2}}{2 \bar{c}_{l}}\right)^{\frac{3}{4}}-\frac{\bar{\vartheta}_{2}}{n}\left(\sum_{l=1}^{n} \frac{\varsigma_{l}^{2}}{2}\right)^{2} \\
& +\left(\sum_{l=1}^{n} \frac{\bar{r}_{l} \tilde{\Theta}_{l}^{2}}{2 \bar{c}_{l}}\right)^{\frac{3}{4}}-\sum_{l=1}^{n} \frac{\bar{r}_{l} \tilde{\Theta}_{l}^{2}}{2 \bar{c}_{l}}+\sum_{l=1}^{n} \frac{\bar{r}_{l} \Theta_{l}^{2}}{2 \bar{c}_{l}}+\sum_{l=1}^{k} \frac{\xi_{l}}{\bar{c}_{l}^{2}} \tilde{\Theta}_{l} \hat{\Theta}_{l}^{3}+\chi_{n} .
\end{aligned}
$$

By utilizing Lemma 3 and making $x_{1}=1, y_{2}=\sum_{l=1}^{n} \frac{\bar{r}_{l} \tilde{\Theta}_{l}^{2}}{2 \bar{c}_{l}}, k_{1}=1-\tau, k_{2}=$ $\tau, \mathrm{B}=e^{(\tau /(1-\tau)) I n \tau}$, it can be acquired

$$
\left(\sum_{l=1}^{n} \frac{\bar{r}_{l} \tilde{\Theta}_{l}^{2}}{2 \bar{c}_{l}}\right)^{\tau} \leq \mathrm{M}(\tau)+\sum_{l=1}^{n} \frac{\bar{r}_{l} \tilde{\Theta}_{l}^{2}}{2 \bar{c}_{l}}
$$

where $\mathrm{M}(\tau)=(1-\tau) \tau^{\frac{\tau}{1-\tau}}$. Set $\tau$ to $\frac{3}{4},(55)$ turn out that

$$
\left(\sum_{l=1}^{n} \frac{\bar{r}_{l} \tilde{\Theta}_{l}^{2}}{2 \bar{c}_{l}}\right)^{\frac{3}{4}} \leq \mathrm{M}(\tau)+\sum_{l=1}^{n} \frac{\bar{r}_{l} \tilde{\Theta}_{l}^{2}}{2 \bar{c}_{l}},
$$

where $\tau_{1}=\mathrm{M}\left(\frac{3}{4}\right)=0.11>0$. 
By substituting (56) into (54), it can be described as

$$
\dot{V}_{n} \leq-\bar{\vartheta}_{1}\left(\sum_{l=1}^{n} \frac{\varsigma_{l}^{2}}{2}\right)^{\frac{3}{4}}-\left(\sum_{l=1}^{n} \frac{\bar{r}_{l} \tilde{\Theta}_{l}^{2}}{2 \bar{c}_{l}}\right)^{\frac{3}{4}} \frac{\bar{\vartheta}_{2}}{n}\left(\sum_{l=1}^{n} \frac{\varsigma_{l}^{2}}{2}\right)^{2}+\sum_{l=1}^{n} \frac{\xi_{l}}{\bar{c}_{l}^{2}} \tilde{\Theta}_{l} \hat{\Theta}_{l}^{3}+\aleph
$$

where $\aleph=\sum_{l=1}^{n} \frac{\bar{r}_{l} \Theta_{l}^{2}}{2 \bar{c}_{l}}+\chi_{n}+\tau_{1}$.

Based on $\tilde{\Theta}_{l} \hat{\Theta}_{l}^{3}=\tilde{\Theta}_{l}\left(\Theta_{l}^{3}-3 \Theta_{l}^{2} \tilde{\Theta}_{l}+3 \Theta_{l} \tilde{\Theta}_{l}^{2}-\tilde{\Theta}_{l}^{3}\right),(57)$ can be obtained

$$
\begin{aligned}
\dot{V}_{n} \leq & -\bar{\vartheta}_{1}\left(\sum_{l=1}^{n} \frac{\varsigma_{l}^{2}}{2}\right)^{\frac{3}{4}}-\left(\sum_{l=1}^{n} \frac{\bar{r}_{l} \tilde{\Theta}_{l}^{2}}{2 \bar{c}_{l}}\right)^{\frac{3}{4}}-\frac{\bar{\vartheta}_{2}}{n}\left(\sum_{l=1}^{n} \frac{\varsigma_{l}^{2}}{2}\right)^{2}+\sum_{l=1}^{n} \frac{3 \xi_{l}}{\bar{c}_{l}^{2}} \tilde{\Theta}_{l}^{3} \Theta_{l} \\
& -\sum_{l=1}^{n} \frac{3 \xi_{l}}{\bar{c}_{l}^{2}} \tilde{\Theta}_{l}^{2} \Theta_{l}^{2}+\sum_{l=1}^{n} \frac{\xi_{l}}{\bar{c}_{l}^{2}} \tilde{\Theta}_{l} \Theta_{l}^{3}-\sum_{l=1}^{n} \frac{\xi_{l}}{\bar{c}_{l}^{2}} \tilde{\Theta}_{l}^{4}+\aleph
\end{aligned}
$$

By utilizing Young's inequality, it can be express as

$$
\begin{gathered}
\sum_{l=1}^{n} \frac{3 \xi_{l}}{\bar{c}_{l}^{2}} \tilde{\Theta}_{l}^{3} \Theta_{l} \leq \sum_{l=1}^{n} \frac{9 \xi_{l} \nu^{\frac{3}{4}}}{4 \bar{c}_{l}^{2}} \tilde{\Theta}_{l}^{4}+\sum_{l=1}^{n} \frac{3 \xi_{l}}{4 \nu^{4} \bar{c}_{l}^{2}} \Theta_{l}^{4}, \\
\sum_{l=1}^{n} \frac{\xi_{l}}{\bar{c}_{l}^{2}} \tilde{\Theta}_{l} \Theta_{l}^{3} \leq \sum_{l=1}^{n} \frac{3 \xi_{l}}{\bar{c}_{l}^{2}} \tilde{\Theta}_{l}^{2} \Theta_{l}^{2}+\sum_{l=1}^{n} \frac{\xi_{l}}{12 \bar{c}_{l}^{2}} \Theta_{l}^{4} .
\end{gathered}
$$

By substituting (59)-(60) into (58), yields

$$
\begin{aligned}
\dot{V}_{n} \leq & -\bar{\vartheta}_{1}\left(\sum_{l=1}^{n} \frac{\varsigma_{l}^{2}}{2}\right)^{\frac{3}{4}}-\left(\sum_{l=1}^{n} \frac{\bar{r}_{l} \tilde{\Theta}_{l}^{2}}{2 \bar{c}_{l}}\right)^{\frac{3}{4}}-\frac{\bar{\vartheta}_{2}}{n}\left(\sum_{l=1}^{n} \frac{\varsigma_{l}^{2}}{2}\right)^{2} \\
& -\sum_{l=1}^{n}\left(4 \xi_{l}-9 \xi_{l} \nu^{\frac{3}{4}}\right)\left(\frac{\tilde{\Theta}_{l}^{2}}{2 \bar{c}_{l}}\right)^{2}+\bar{\aleph}
\end{aligned}
$$

where $\bar{\aleph}=\sum_{l=1}^{n} \frac{\xi_{l}}{12 \bar{c}_{l}^{2}} \Theta_{l}^{4}+\sum_{l=1}^{n} \frac{3 \xi_{l}}{4 \nu^{4} \bar{c}_{l}^{2}} \Theta_{l}^{4}+\aleph$.

Thereby, (61) can be rewritten as

$$
\begin{aligned}
\dot{V}_{n} \leq & -\bar{\vartheta}_{1}\left(\sum_{l=1}^{n} \frac{\varsigma_{l}^{2}}{2}\right)^{\frac{3}{4}}-\hat{\vartheta}_{1}\left(\sum_{l=1}^{n} \frac{\tilde{\Theta}_{l}^{2}}{2 \bar{c}_{l}}\right)^{\frac{3}{4}} \\
& -\frac{\bar{\vartheta}_{2}}{n}\left(\sum_{l=1}^{n} \frac{\varsigma_{l}^{2}}{2}\right)^{2}-\frac{\hat{\vartheta}_{2}}{n}\left(\sum_{l=1}^{n} \frac{\tilde{\Theta}_{l}^{2}}{2 \bar{c}_{l}}\right)^{2}+\bar{\aleph},
\end{aligned}
$$

where $\hat{\vartheta}_{1}=\min \left\{\bar{r}_{l}\right\}$ and $\hat{\vartheta}_{2}=\min \left\{4 \xi_{l}-9 \xi_{l} \nu^{\frac{3}{4}}\right\}$ 
By defining $\vartheta_{1}=\min \left\{\bar{\vartheta}_{1}, \hat{\vartheta}_{1}\right\}, \vartheta_{2}=\min \left\{\frac{\bar{\vartheta}_{2}}{n}, \frac{\hat{\vartheta}_{2}}{n}\right\},(62)$ can be shown as

$$
\begin{aligned}
\dot{V}_{n} \leq & -\vartheta_{1}\left\{\left(\sum_{l=1}^{n} \frac{\tilde{\Theta}_{l}^{2}}{2 \bar{c}_{l}}\right)^{\frac{3}{4}}+\left(\sum_{l=1}^{n} \frac{\varsigma_{l}^{2}}{2}\right)^{\frac{3}{4}}\right\} \\
& -\vartheta_{2}\left\{\left(\sum_{l=1}^{n} \frac{\varsigma_{l}^{2}}{2}\right)^{2}+\left(\sum_{l=1}^{n} \frac{\tilde{\Theta}_{l}^{2}}{2 \bar{c}_{l}}\right)^{2}\right\}+\bar{\aleph} .
\end{aligned}
$$

As we all know that

$$
V_{n}=\sum_{l=1}^{n} \frac{\varsigma_{l}^{2}}{2}+\sum_{l=1}^{n} \frac{\tilde{\Theta}_{l}^{2}}{2 \bar{c}_{l}} .
$$

Furthermore, it follows from Lemma 4 and Lemma 5 that

$$
\begin{gathered}
V_{n}^{2} \leq 2 n\left\{\left(\sum_{l=1}^{n} \frac{\varsigma_{l}^{2}}{2}\right)^{2}+\left(\sum_{l=1}^{n} \frac{\tilde{\Theta}_{l}^{2}}{2 \bar{c}_{l}}\right)^{2}\right\}, \\
V_{n}^{\frac{3}{4}} \leq\left(\sum_{l=1}^{n} \frac{\varsigma_{l}^{2}}{2}\right)^{\frac{3}{4}}+\left(\sum_{l=1}^{n} \frac{\tilde{\Theta}_{l}^{2}}{2 \bar{c}_{l}}\right)^{\frac{3}{4}} .
\end{gathered}
$$

By combining (65) with $(66,(63)$ gives

$$
\dot{V}_{n} \leq-\vartheta_{1} V_{n}^{\frac{3}{4}}-\frac{\vartheta_{2}}{2 n} V_{n}^{2}+\bar{\aleph} .
$$

Until now, the FODSC-based fixed-time adaptive control problem has been completed.

\subsection{Stability Analysis}

Based on the above analysis, stability result can be summarized as the following theorem.

Theorem 1. Consider the strict-feedback system (1) under Assumptions $1-2$. If the virtual control signals (25), (35) and (46), the adaptive updating laws (26), (36) and (47), the error compensation signals (16), and the actual control signal (2)-(4), then the following properties hold:

- All the signals in the CLS are practically fixed-time bounded.

- The tracking errors can be regulated to predefined boundary in fixedtime.

- The Zeno behavior can be excluded.

Proof. For the auxiliary system (16), we prove that $\eta_{l}$ can be bounded in the fixed-time $T_{1}$ by the following step. 
Step $n+1$. Consider the Lyapunov function $\bar{V}_{\eta}=\sum_{l=1}^{n} \frac{1}{2} \eta_{l}^{2}$ and its derivative as follows:

$$
\begin{aligned}
\dot{\bar{V}}_{\eta}= & -k_{11} \eta_{1}^{\frac{3}{2}}-k_{12} \eta_{1}^{4}+d_{1} \eta_{1} \eta_{2}+d_{1} \eta_{1}\left(\alpha_{2}^{*}-\alpha_{2}\right) \\
& -k_{21} \eta_{2}^{\frac{3}{2}}-k_{22} \eta_{2}^{4}-d_{1} \eta_{1} \eta_{2}+\eta_{2} \eta_{3}+\eta_{2}\left(\alpha_{3}^{*}-\alpha_{3}\right) \\
& -k_{31} \eta_{3}^{\frac{3}{2}}-k_{32} \eta_{3}^{4}-\eta_{2} \eta_{3}+\eta_{3} \eta_{4}+\eta_{3}\left(\alpha_{4}^{*}-\alpha_{4}\right) \\
& +\cdots \\
& -k_{n 1} \eta_{n}^{\frac{3}{2}}-k_{n 2} \eta_{n}^{4}-\eta_{n-1} \eta_{n} \\
= & -\sum_{l=1}^{n} k_{l 1} \eta_{l}^{\frac{3}{2}}+\sum_{l=1}^{n-1} d_{l} \eta_{l}\left(\alpha_{l+1}^{*}-\alpha_{l+1}\right)-\sum_{l=1}^{n} k_{l 2} \eta_{l}^{4}
\end{aligned}
$$

By utilizing the Lemma 4 and Lemma 5 in [15], $\left|\alpha_{l+1}^{*}-\alpha_{l+1}\right| \leq \Delta_{l}$ can be obtained in a fixed-time $T_{2}$. Thus, (68) yields

$$
\begin{aligned}
\dot{\bar{V}}_{\eta} & \leq-\sum_{l=1}^{n} k_{l 1} \eta_{l}^{\frac{3}{2}}-\sum_{l=1}^{n} k_{l 2} \eta_{l}^{4}+\left|d_{n}\right|\left|\eta_{n}\right| \partial_{n}+\sum_{l=1}^{n-1}\left|d_{l}\right|\left|\eta_{l}\right|\left|\alpha_{l+1}^{*}-\alpha_{l+1}\right| \\
& \leq-\sum_{l=1}^{n} k_{l 1} \eta_{l}^{\frac{3}{2}}-\sum_{l=1}^{n} k_{l 2} \eta_{l}^{4}+\frac{1}{2} \sum_{l=1}^{n} \eta_{l}^{2}+\sum_{l=1}^{n} \frac{\Delta_{l}}{2}
\end{aligned}
$$

Due to the fact that $\eta_{l}^{2} \leq\left(\eta_{l}^{2}\right)^{\frac{1}{2}}+\left(\eta_{l}^{2}\right)^{3}$, one has

$$
\frac{1}{2} \sum_{l=1}^{n} \eta_{l}^{2} \leq \frac{1}{2} \sum_{l=1}^{n}\left(\left(\eta_{l}^{2}\right)^{\frac{3}{4}}+\left(\eta_{l}^{2}\right)^{2}\right) .
$$

By combining (70) with Lemma 5, (69) can be indicated as

$$
\begin{aligned}
\dot{\bar{V}}_{\eta} & \leq-\sum_{l=1}^{n}\left(k_{l 1}-\frac{1}{2}\right) \eta_{l}^{\frac{3}{2}}-\sum_{l=1}^{n}\left(k_{l 2}-\frac{1}{2}\right) \eta_{l}^{4}+\sum_{l=1}^{n} \frac{\Delta_{l}}{2} \\
& \leq-\lambda_{1} V_{\eta}^{\frac{3}{4}}-\lambda_{2} V_{\eta}^{2}+\bar{\kappa},
\end{aligned}
$$

where $\lambda_{1}=\min \left\{2^{\frac{3}{4}}\left(k_{n 1}-\frac{1}{2}\right)\right\}, \lambda_{2}=\left\{2^{2} n^{-1}\left(k_{n 2}-\frac{1}{2}\right)\right\}$ and $\bar{\kappa}=\sum_{l=1}^{n} \frac{\Delta_{l}}{2}$. Thus, it can be obtained that $\eta_{l}$ is practically fixed-time bounded in $T_{1}$. This completes the proof.

According to (67) and Lemma 1, the fixed-time convergence problem was considered as follows:

$$
\dot{V}_{n} \leq-\vartheta_{1} V_{n}^{\frac{3}{4}}-(1-l) \frac{\vartheta_{2}}{2 n} V_{n}^{2}-l \frac{\vartheta_{2}}{2 n} V_{n}^{2}+\bar{\aleph},
$$

where $0<l<1$ and $(72)$ can be rewritten as $\dot{V}_{n} \leq-\vartheta_{1} V_{n}^{\frac{3}{4}}-(1-l) \frac{\vartheta_{2}}{2 n} V_{n}^{2}$, if $V_{n}^{2}>\frac{2 n \bar{\aleph}}{l \vartheta_{2}}$. The setting time of the CLS in fixed-time is expressed as follows:

$$
T_{3} \leq \frac{4}{\vartheta_{1}}+\frac{2 n}{\vartheta_{2}(1-l)}
$$


In view of $V_{n}^{2} \leq \frac{2 n \bar{\aleph}}{l \vartheta_{2}}$, it can be concluded that $V_{n}$ is bounded, and then $\varsigma_{i}, \tilde{\Theta}_{i}, 1 \leq i \leq n$, are also bounded, so does $z_{i}, m_{i}, j_{i}$. In view of $-\xi<j_{1}<\xi$, the prescribed performance is guaranteed effectively. Moreover, it follows from the boundedness of $\varsigma_{i}, z_{i}, \eta_{i}$ that $\alpha_{i}^{*}, \alpha_{i}$ is also bounded. Since $z_{i}=x_{i}-\alpha_{i}^{*}$, all the state variables $x_{i}$ are all bounded. Therefore, all signals in the CLS are bounded within the setting time $T_{l}=T_{1}+T_{2}+T_{3}$.

Furthermore, it can be confirmed that all signals are bounded, i.e., $\dot{v}$ is a function of all the bounded signals. There is a constant $c$ satisfying $|\dot{v}| \leq c$. Note that $\mathrm{P}\left(t_{k}\right)=0 \quad\left(\mathrm{P}\left(t_{k}\right)=v\left(t_{k}\right)-u\left(t_{k}\right)\right)$ and $\lim _{t \rightarrow t_{k+1}} \mathrm{P}=\tau|u|+\mu_{2}$, then inter-execution intervals $t_{k}-t_{k+1} \geq \frac{\tau|u|+\mu_{2}}{c}$. Thus, it can be obtained that the inter-execution intervals $t^{*}$ satisfiy ${t^{*}}^{c}=\frac{\mu_{2}}{c}>0$, in other words, excluding the Zeno-behavior. The proof is completed.

\section{SIMULATION}

Two examples including a comparative example and a practical example are provided to testify the feasibility and superiority of the proposed control strategy in this section.

Numerical Example: Consider the following second-order nonlinear system is adopted in [29]:

$$
\left\{\begin{array}{l}
\dot{x}_{1}=x_{2}+0.5 x_{1}^{2} \\
\dot{x}_{2}=u+0.5 x_{1} x_{2} \sin \left(x_{1}\right) \\
y=x_{1}
\end{array}\right.
$$

where the reference trajectory is designed as $y_{d}=0.5 \sin t$ and the following performance function is given

$$
\sigma=\left\{\begin{array}{l}
(0.45-0.45 t)^{3}+0.015,0 \leq t<1 \\
0.015, t \geq 1
\end{array}\right.
$$

To emphasize the contribution of this paper, the virtual control signals are set up as

$$
\begin{aligned}
\alpha_{2}= & -\frac{d_{1}}{2 a_{11}} \hat{\Theta}_{1}\left\|S_{1}\right\|^{2} \varsigma_{1}-\frac{k_{11}}{d_{1}} z_{1}^{\frac{1}{2}}-\frac{k_{12}}{d_{1}} z_{1}^{3}-\frac{1}{2 a_{12}} d_{1} \varsigma_{1} \\
& +\dot{y}_{d}+\frac{2}{\pi} \dot{\xi} \arctan \left(m_{1}\right) \\
\alpha_{3}= & -\frac{1}{2 a_{21}} \hat{\Theta}_{2}\left\|S_{2}\right\|^{2} \varsigma_{2}-k_{21} z_{2}^{\frac{1}{2}}-k_{22} z_{2}^{3}-\frac{1}{2 a_{22}} \varsigma_{2}-d_{1} \eta_{1} .
\end{aligned}
$$

The adaptive laws are chosen as

$$
\begin{aligned}
& \dot{\hat{\Theta}}_{1}=\frac{d_{1}^{2} \bar{c}_{1}}{2 a_{11}}\left\|S_{1}\right\|^{2} \varsigma_{1}^{2}-\bar{r}_{1} \hat{\Theta}_{1}-\frac{\xi_{1}}{\bar{c}_{1}} \hat{\Theta}_{1}^{3} . \\
& \dot{\hat{\Theta}}_{2}=\frac{\bar{c}_{2}}{2 a_{21}}\left\|S_{2}\right\|^{2} \varsigma_{2}^{2}-\bar{r}_{2} \hat{\Theta}_{2}-\frac{\xi_{2}}{\bar{c}_{2}} \hat{\Theta}_{2}^{3} .
\end{aligned}
$$




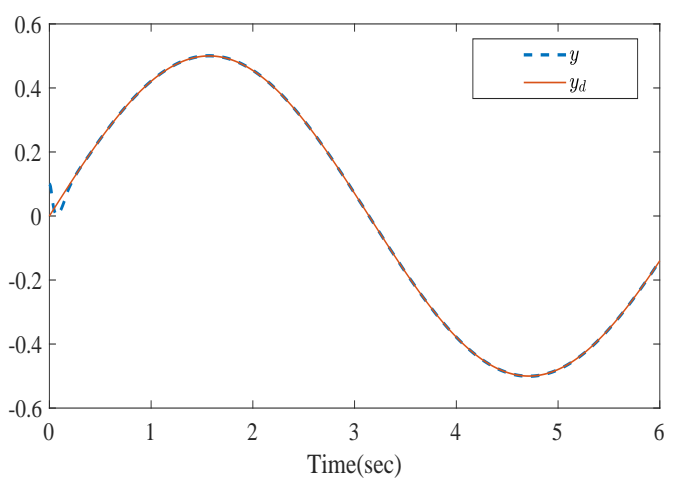

Fig. 2: The curves of $y$ and $y_{d}$

The relative-threshold-based control signals are constructed in (2)-(4), and the compensating signal is defined as in (16).

The initial values are chosen as $x_{1}(0)=0.1, x_{2}(0)=-0.1, \hat{\Theta}_{1}(0)=$ $0, \hat{\Theta}_{2}(0)=0.5$ and the corresponding design parameters are chosen as $\rho=$ $1, K_{11}=K_{12}=5, K_{21}=K_{22}=50, a_{11}=a_{12}=a_{21}=a_{22}=0.5, \mu_{1}=2, \mu_{2}=$ $0.1, \tau=0.5, \beta=0.05, \bar{c}_{1}=\bar{c}_{2}=1, \xi_{1}=\xi_{2}=5, \bar{r}_{1}=\bar{r}_{2}=5, q=0.9$.

To facilitate controller design, two RBFNNs are adopted to handle the unknown terms $\Lambda_{1}=f_{1}\left(x_{1}\right)$ and $\Lambda_{2}=f_{2}(x)-\dot{\alpha}_{2}^{*}+d_{1} \varsigma_{1}$ existing in the nonlinear systems. The basis function vector $S_{i}\left(z_{i}\right)=\left[s_{i 1}\left(z_{i}\right), \ldots, s_{i m_{i}}\left(z_{i}\right)\right]^{T} \in R^{m_{i}}$ can be obtained by the Gaussian function $\left.\exp \left[-\left(\left(z_{i}-c_{i j}\right)^{T}\left(z_{i}-c_{i j}\right)\right) / b_{i j}^{2}\right)\right],(i=$ 1,$\left.2 ; j=1, \ldots, m_{i}\right)$ with $m_{i}=9, c_{i j}=(j-5)[1,1,1]^{T}, j=1, \ldots, m_{i}$. The input vectors of RBFNNs and the width of Gaussian functions are chosen as $z_{i}=\left[x_{1}, x_{2}, D^{q} \alpha_{2}^{*}\right]^{\mathrm{T}}$ and $b_{i j}=1\left(i=1,2 ; j=1, \ldots, m_{i}\right)$, respectively.

Validity analysis: Fig. 2 shows the trajectory of output signal $y$ and the target signal $y_{d}$. Fig. 3 plots the tracking error can strictly converge into prescribed boundaries within fixed-time. From Figs. 2-3, it can be seen that our proposed scheme has better performance accuracy. Furthermore, the trajectories of the adaptive parameters $\hat{\Theta}_{1}, \hat{\Theta}_{2}$ are depicted in Fig. 4, the control signal $u$ is shown in Fig. 5. Obviously, it can be observed from Figs. 2-5 that all signals in the resulting CLS are bounded in fixed-time interval.

Comparative analysis: To achieve an intuitive comparison, the design parameters in the paper are selected as same as the reference [29]. Compared with the simulation results in [29], it can be easily observed that the proposed control method provides a faster convergence time, while the tracking error can more quickly converge toward predefined range. Therefore, our control strategy is superior than that proposed in [29] for accommodating better performance requirements. 


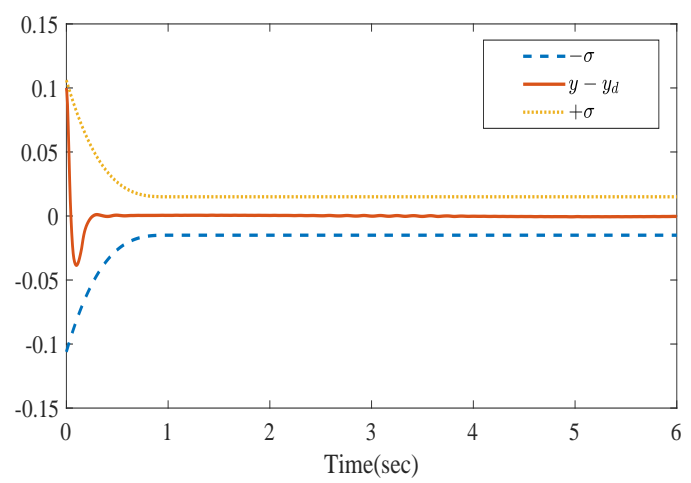

Fig. 3: The tracking error $y-y_{d}$

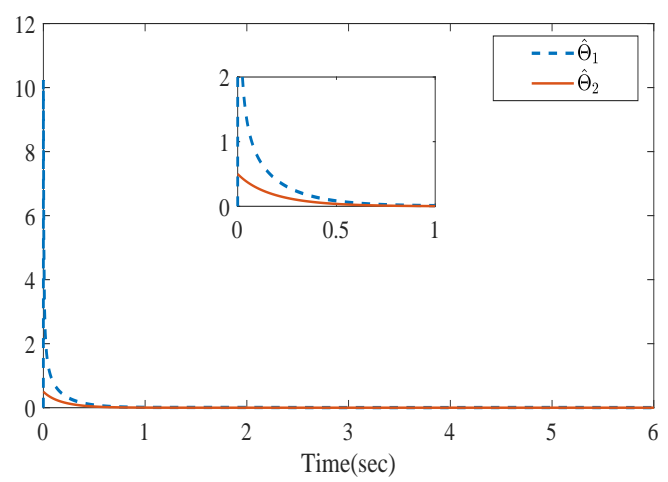

Fig. 4: The adaptive parameter $\hat{\Theta}_{1}$ and $\hat{\Theta}_{2}$

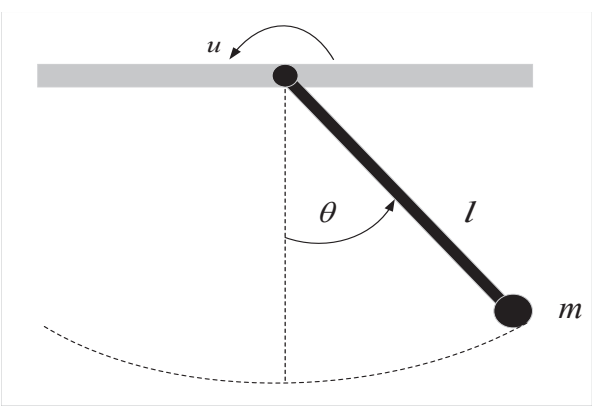

Fig. 6: The pendulum model. 


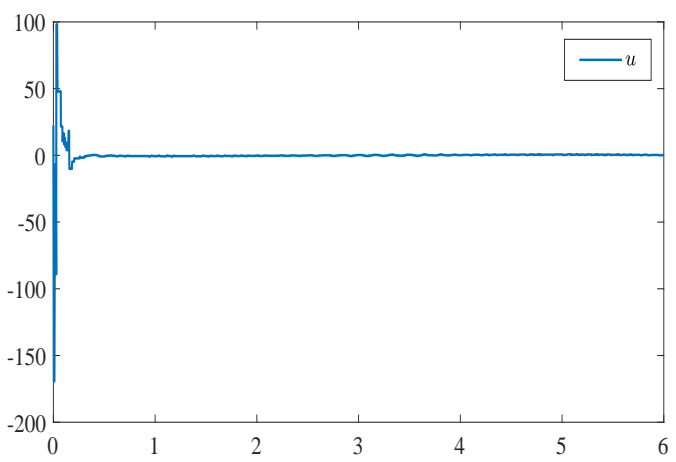

Fig. 5: The control signal $u$

Practical Example: The dynamics of the pendulum model is

$$
m l \ddot{\phi}+k l \dot{\phi}+m g \sin \phi=u,
$$

where $\phi$ denotes the angle, $\dot{\phi}$ denotes the angular velocity, $l$ denotes the length of the pendulum, $m$ denotes the mass of the pendulum, $k$ denotes an unknown frictional index, $g$ denotes the acceleration of gravity.

Consider $x_{1}=m l \phi$ and $x_{2}=m l \dot{\phi},(73)$ can be rewritten as

$$
\left\{\begin{array}{l}
\dot{x}_{1}=x_{2}, \\
x_{2}=u-m g \sin \left(\frac{x_{1}}{m l}\right)-\frac{k}{m} x_{2}, \\
y=x_{1} .
\end{array}\right.
$$

The target signal is chosen as $y_{d}=0.5(\sin (t)+\sin (0.5 t))$, the RBFNN membership functions and the desired performance function are selected as in Example 1.

The original values of the pendulum are defined as $x_{1}(0)=0.1, x_{2}(0)=$ $-0.1, \hat{\Theta}_{1}(0)=0, \hat{\Theta}_{2}(0)=0.5$. The relevant parameters are selected as $k=$ 1.095, $m=1, l=1, K_{11}=K_{12}=5, K_{21}=K_{22}=50, a_{11}=a_{12}=a_{21}=a_{22}=$ $0.5, \bar{c}_{1}=\bar{c}_{2}=1, \xi_{1}=\xi_{2}=5, \bar{r}_{1}=\bar{r}_{2}=5, \mu_{1}=2, \mu_{2}=0.1, \rho=1, \tau=0.5, \beta=$ $0.05, q=0.9$

The pendulum plant is shown in Fig. 6 and the relative results are described in Figs. 7-10, where Fig. 7 plots the responses of output signal $y$ and tracking signal $y_{d}$. The profile of tracking error is illustrated in Fig. 8. By contrast, it has better tracking accuracy than [29]. Fig. 9 shows the responses of the adaptive parameters $\hat{\Theta}_{1}, \hat{\Theta}_{2}$ and the trajectory of the control signal $u(t)$ is displayed in Fig. 10. Furthermore, it can be inferred that the proposed method can guarantee that all the signals of the resulting CLS are bounded in fixed-time and the system output can track the target signal with an ideal approximation accuracy. 


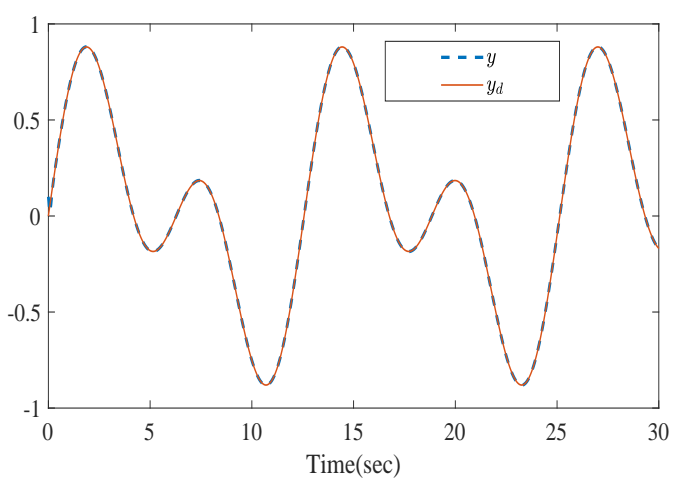

Fig. 7: The trajectories of $y$ and $y_{d}$

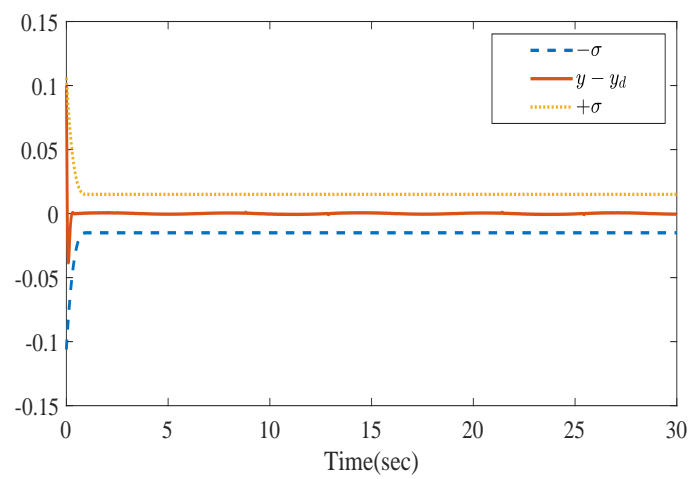

Fig. 8: The tracking error $y-y_{d}$

\section{Conclusions}

In this article, an event-based adaptive NN fixed-time control problem for strict-feedback nonlinear systems with prescribed performance has been studied. Based on the FODSC technique, by combining the approximation ability of $\mathrm{NN}$ and the command filter backstepping method, the unknown nonlinear functions and the issue of "explosion of complexity" have been conquered, respectively. Meanwhile, the novel error compensation signal is established for filter error and an event-triggered controller has been developed to save the transmission resources, where is no Zeno phenomenon. Furthermore, by applying the performance function to ensure the tracking error converges into predefined bounds in the fixed-time interval. Finally, comparative and prac- 


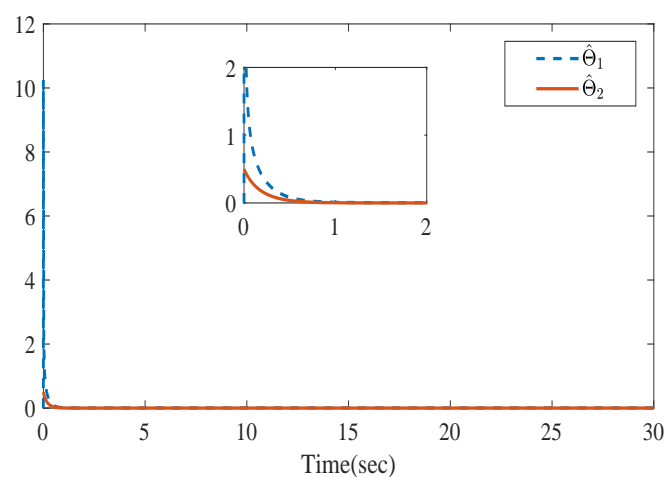

Fig. 9: The adaptive parameter $\hat{\Theta}_{1}$ and $\hat{\Theta}_{2}$

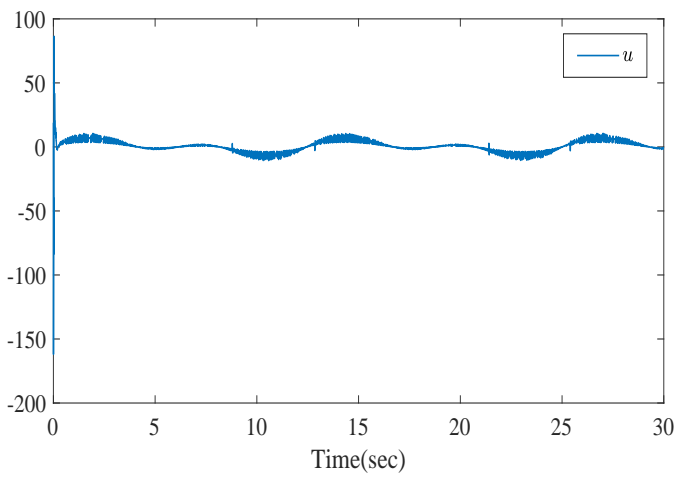

Fig. 10: Control signal $u$

tical examples are presented to confirm the feasibility and superiority of the proposed control method.

\section{Funding}

This work was supported by the National Natural Science Foundation of China under Grant 61976081, the Natural Science Fund for Excellent Young Scholars of Henan Province under grant 202300410127. 


\section{Conflict of Interest}

The authors have no conflicts of interest to declare that are relevant to the content of this article.

\section{Data Availability Statements}

All data generated or analysed during this study are included in this published article (and its supplementary information files).

\section{References}

1. Federico, B.A., Krstic, M.: Backstepping-forwarding control and observation for hyperbolic PDEs with fredholm integrals.IEEE Trans. Autom. Control 60(8), 2145-2160 (2015)

2. Ma, H., Liang, H.J., Zhou, Q., Ahn, C.K.: Adaptive dynamic surface control design for uncertain nonlinear strict-feedback systems with unknown control direction and disturbances. IEEE Trans. Syst. Man Cybernet. Syst. 49(3), 506-515 (2018)

3. Ma, H.F., Xu, S.Y., Zhang, B.Y., Ma, Q.: Globally adaptive control for stochastic nonlinear time-delay systems with perturbations and its application. Automatica. 102, 105-110 (2019)

4. Wu, L.B., Park, J.H., Xie, X.P., Ren, Y.W., Yang, Z.C.: Distributed adaptive neural network consensus for a class of uncertain nonaffine nonlinear multi-agent systems. Nonlin. Dynam. 100(2), 1243-1255 (2020)

5. Song, S., Zhang, B.Y., Xia, J.W., Zhang, Z.Q.: Adaptive backstepping hybrid fuzzy sliding mode control for uncertain fractional-order nonlinear systems based on finite-time scheme. IEEE Trans. Syst. Man Cybernet. Syst. 50(4), 1559-1569 (2020)

6. Li, S., Ahn, C.K., Chadli, M., Xiang, Z.R.: Sampled-data adaptive fuzzy control of switched large-scale nonlinear delay systems. IEEE Trans. Fuzzy Syst. (2021). Doi: 10.1109/TFUZZ.2021.3052094

7. Ma, L., Huo, X., Zhao, X.D., Zong, G.D.: Observer-based adaptive neural tracking control for output-constrained switched MIMO nonstrict-feedback nonlinear systems with unknown dead zone. Nonlin. Dynam. 99(2), 1019-1036 (2020)

8. Niu, B., Wang, D., Alotaibi, N.D., Alsaadi, F.E.: Adaptive neural state-feedback tracking control of stochastic nonlinear switched systems: An average dwell-time method. IEEE Trans. Neural Netw. Learn. Syst. 30(4), 1076-1087 (2018)

9. Yoo S.J.: Neural-network-based adaptive resilient dynamic surface control against unknown deception attacks of uncertain nonlinear time-delay cyberphysical systems. IEEE Trans. Neural Netw. Learn. Syst. 31(10), 4341-4353 (2019)

10. Farrell, J.A., Polycarpou, M., Sharma, M., Dong, W.J.: Command filtered backstepping. IEEE Trans. Autom. Control 54(6), 1391-1395 (2009)

11. Dong, W.J., Farrell, J.A., Polycarpou, M.M., Djapic, V., Sharma, M.: Command filtered adaptive backstepping. IEEE Trans. Control Syst. Technol. 20(3), 566-580 (2011)

12. Ma, J.L., Park, J.H., Xu, S.Y., Cui, G.Z, Yang, Z.Y.: Command-filter-based adaptive tracking control for nonlinear systems with unknown input quantization and mismatching disturbances. Applied Mathema. Computa. 377, 125161 (2020)

13. Wang, H.Q., Kang, S.J., Zhao, X.D., Xu, N., Li, T.S.: Command Filter-Based Adaptive Neural Control Design for Nonstrict-Feedback Nonlinear Systems With Multiple Actuator Constraints. IEEE Trans. Cybern. (2021) Doi: 10.1109/TCYB.2021.3079129

14. Li, M., Li, S., Ahn, C.K., Xiang, Z.R.: Adaptive fuzzy event-triggered command-filtered control for nonlinear time-delay systems. IEEE Trans. Fuzzy Syst. (2021). Doi: 10.1109/TFUZZ.2021.3052095

15. Yu, J.P., Shi, P., Zhao, L.: Finite-time command filtered backstepping control for a class of nonlinear systems. Automatica 92, 173-180 (2018) 
16. Xia, J.W., Li, B.M., Su, S.F., Sun, W., Shen, H.: Finite-time command filtered eventtriggered adaptive fuzzy tracking control for stochastic nonlinear systems. IEEE Trans. Fuzzy Syst. 29(7), 1815-1825 (2021)

17. Li, Y.X., Hou, Z.S., Che, W.W., Wu, Z.G.: Event-based design of finite-time adaptive control of uncertain nonlinear systems. IEEE Trans. Neural Netw. Learn. Syst. (2021) Doi: $10.1109 /$ TNNLS.2021.3054579

18. Polyakov, A.: Nonlinear feedback design for fixed-time stabilization of linear control systems. IEEE Trans. Autom. Control 57(8), 2106-2110 (2011)

19. Chen, M., Wang, H.Q., Liu, X.P.: Adaptive fuzzy practical fixed-time tracking control of nonlinear systems. IEEE Trans. Fuzzy Syst. 29(3), 664-673 (2021)

20. Ling, S., Wang, H.Q., Liu, P.X.: Fixed-time adaptive event-triggered tracking control of uncertain nonlinear systems. Nonlin. Dynam. 100(1), 3381-3397 (2020)

21. Shi, W.X.: Adaptive Fuzzy Output-Feedback Control for Nonaffine MIMO Nonlinear Systems With Prescribed Performance. IEEE Trans. Fuzzy Syst. 29(5), 1107-1120 (2020)

22. Li, Y.M., Shao, X.F., Tong, S.C.: Adaptive fuzzy prescribed performance control of nontriangular structure nonlinear systems. IEEE Trans. Fuzzy Syst. 28(10), 2416-2426 (2019)

23. Sui, S., Chen, C.P., Tong, S.C.: Finite-Time Adaptive Fuzzy Prescribed Performance Control for High-Order Stochastic Nonlinear Systems. IEEE Trans. Fuzzy Syst. (2021). Doi: 10.1109 /TFUZZ.2021.3077317

24. Zhou, Q., Zhao, S.Y., Li, H.Y., Lu, R.Q., Wu, C.W.: Adaptive neural network tracking control for robotic manipulators with dead zone. IEEE Trans. Neural Netw. Learn. Syst. 30(12), 3611-3620 (2018)

25. Xie, W.B., Guo, M.H., Xu, B.L, Wang, X.: Fault tolerant robust control with transients for over-actuated nonlinear systems. Nonlin. Dynam. 104(3), 2433-2450 (2021)

26. Yang, C.Y., Xia, J.W., Park, J.H., Shen, H., Wang, J.: Sliding mode control for uncertain active vehicle suspension systems: an event-triggered $H_{\infty}$ control scheme. Nonlin. Dynam. 103(4), 3209-3221 (2021)

27. Liu, L., Liu, Y.J., Tong, S.C., Gao, Z.W.: Relative Threshold-Based Event-Triggered Control for Nonlinear Constrained Systems With Application to Aircraft Wing Rock Motion. IEEE Trans. Industrial Info. (2021). Doi: 10.1109/TII.2021.3080841

28. Sun, K.K., Qiu, J.B., Karimi, H.R., Fu, Y.L.: Event-triggered robust fuzzy adaptive finite-time control of nonlinear systems with prescribed performance. IEEE Trans. Fuzzy Syst. 29(6), 1460-1471 (2020)

29. Sun, W., Wu, Y.Q., Sun, Z.Y.: Command filter-based finite-time adaptive fuzzy control for uncertain nonlinear systems with prescribed performance. IEEE Trans. Fuzzy Syst. 28(12), 3161-3170 (2020)

30. Hua, C.C., Liu, G.P., Li, L., Guan, X.P.: Adaptive fuzzy prescribed performance control for nonlinear switched time-delay systems with unmodeled dynamics. IEEE Trans. Fuzzy Syst. 26(4), 1934-1945 (2017)

31. Zhou, Q., Li, H.Y., Wang, L.J., Lu, R.Q.: Prescribed performance observer-based adaptive fuzzy control for nonstrict-feedback stochastic nonlinear systems. IEEE Trans. Syst. Man Cybernet. Syst. 48(10), 1747-1758 (2017)

32. Li, H.Y., Zhao, S.Y., He, W., Lu, R.Q.: Adaptive finite-time tracking control of full state constrained nonlinear systems with dead-zone. Automatica 100, 99-107 (2019)

33. Podlubny, I.: Fractional Differential Equations. New York, NY, USA: Academic (1998)

34. Jin, X.: Adaptive fixed-time control for MIMO nonlinear systems with asymmetric output constraints using universal barrier functions. IEEE Trans. Autom. Control 64(7), 3046-3053 (2018)

35. Chen, B., Liu, X.P., Liu, K.F., Lin, C.: Novel adaptive neural control design for nonlinear MIMO time-delay systems. Automatica 45(6), 1554-1560 (2009)

36. Qian, C.J., Lin, W.: Non-lipschitz continuous stabilizers for nonlinear systems with uncontrollable unstable linearization. Systems Control Letters 42(3), 185-200 (2001)

37. Zhu, Z., Xia, Y.Q., Fu, M.Y.: Attitude stabilization of rigid spacecraft with finite-time convergence. Int. J. Robust Nonlinear Control 21(6), 686-702 (2011)

38. Hardy, G.H., Littlewood, J.E., Polya, G.: Inequalities. London, U.K.: Cambridge Univ. Press (1952) 
39. Xing, L.T., Wen, C.Y., Liu, Z.T., Su, H.Y., Cai, J.P.: Event-triggered adaptive control for a class of uncertain nonlinear systems. IEEE Trans. Autom. Control 62(4), 2071-2076 (2016)

40. Li, Y., Chen, Y.Q., Podlubuny, I.: MittagLeffler stability of fractional order nonlinear dynamic systems. Automatica 45(8), 1965-1969 (2009)

41. Song, S., Zhang, B.Y., Song, X.N., Zhang, Z.Q.: Neuro-fuzzy-based adaptive dynamic surface control for fractional-order nonlinear strict-feedback systems with input constraint. IEEE Trans. Syst. Man Cybernet. Syst. 51(46), 3575-3586 (2021)

42. Liu, Y., Liu, X.P., Jing, Y.W., Zhang, Z.Y.: A novel finite-time adaptive fuzzy tracking control scheme for nonstrict feedback systems. IEEE Trans. Fuzzy Syst. 27(4), 646-658 (2018) 